\title{
Intra-myometrial pregnancy-A rare site of ectopic pregnancy
}

\author{
Venkatesh M, Sindhuja KLN* and Sundeep NVK \\ Department of Radiodiagnosis, Narayana medical college, Nellore, India
}

\begin{abstract}
Ectopic gestation constitutes 1-2\% of all first trimester pregnancies. Nearly $95 \%$ are of tubal origin and $5 \%$ are non-tubal origin. Intra-myometrial pregnancy constitutes less than $1 \%$ of all the ectopic pregnancies. We present a rare case of interstitial myometrial pregnancy diagnosed confidently on Ultrasound and MRI with active cardiac activity. Diagnosis of the ectopic gestation is very much essential to prevent fatal outcomes.
\end{abstract}

\section{Introduction}

Diagnosis of ectopic pregnancy and appropriate management are very essential in pregnant women to prevent adverse outcomes. Intramyometrial pregnancy is one such rare sub-type of ectopic pregnancy. If not diagnosed and managed, it will result in the rupture of the uterus resulting in increased maternal mortality.

\section{Case report}

A 35-year-old G2 P1 L1 lady presented with amenorrhea of 7 weeks and mild lower abdominal pain. No prior history of abortion or dilatation and curettage. Urine pregnancy test was positive and early antenatal ultrasound was advised. Transvaginal sonography was performed which revealed a gestational sac in the myometrium close to serosa (Figure 1A). Fetal pole was seen within the sac with good fetal cardiac activity (Figure 1B). MRI pelvis was done for better delineation of the sac. MRI (Figure 2A and 2C) showed gestational sac with hypointense fetal pole in the anterior myometrium as revealed on USG. MRI (Figure 2B) revealed additional finding of T2 curvilinear hyperintense tract which was seen extending from the endometrial cavity to gestational sac. Bilateral adnexa were normal and there was no free fluid in pelvis. USG and MRI features were suggestive of intramyometrial pregnancy. In view of location of gestational sac in the myometrium close to serosa with high risk of rupture, wedge resection of myometrium was performed (Figure 2D). Patient was discharged on $5^{\text {th }}$ post-operative day with no complications.

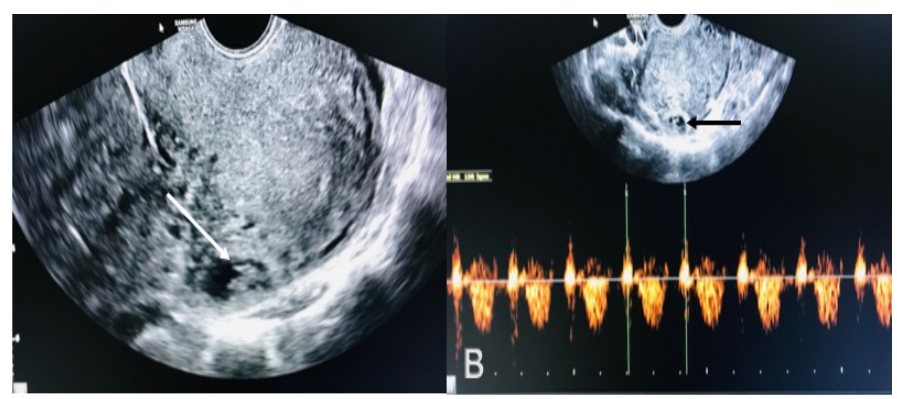

Figure 1A. Transvaginal sonography showing gestational sac with fetal pole in the myometrium(arrow), 1B Colour doppler revealed fetal cardiac activity

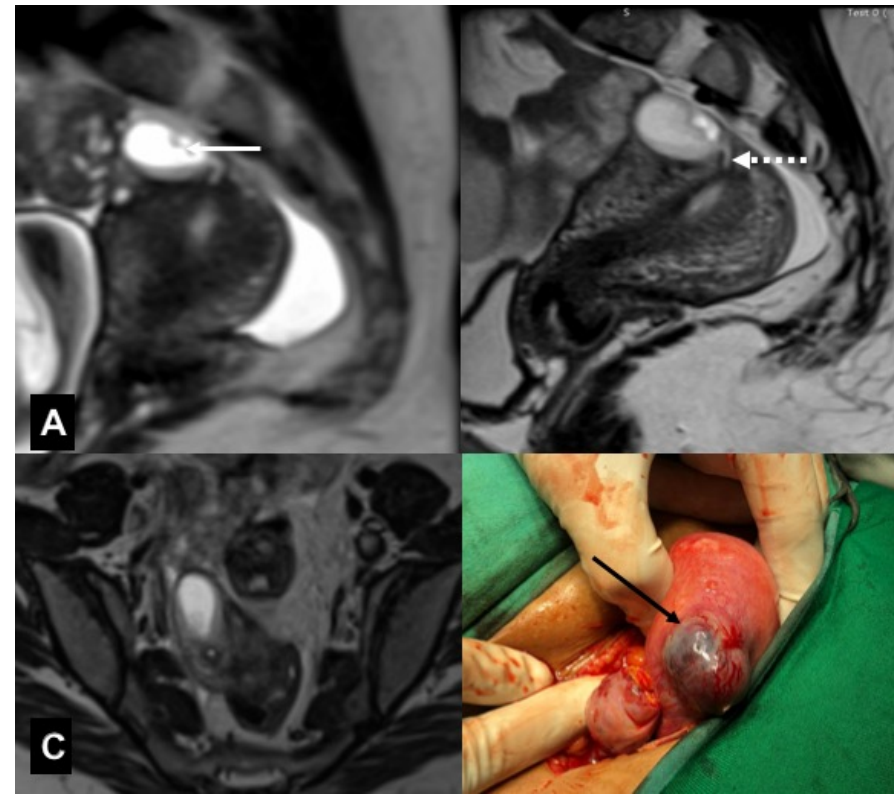

Figure 2. Saggital T2 weighted image of uterus showing $\mathrm{T} 2$ hyperintense gestational sac with hypointense fetal pole $(\mathrm{A}, \mathrm{C})$ in the subserosal plane with small curvilinear hyperintense tract (B-dashed arrow). Intraoperative image of uterus showing focal bulging over the uterus with gestational sac (Black arrow) (D)

\section{Discussion}

Ectopic gestation is the implantation of blastocyst other than the uterine cavity constituting $1-2 \%$ of all the first trimester pregnancies [1]. Among them, nearly $95 \%$ are implanted in various segments of the fallopian tube and rest $5 \%$ in the ovary, cervix, peritoneal

${ }^{\star}$ Correspondence to: Sindhuja KLN, Department of Radiodiagnosis, Narayana medical college, Nellore, India, E-mail: cindrella.kln121@gmail.com

Key words: intra-myometrial pregnancy, myomectomy, dilatation and curettage

Received: February 02, 2020; Accepted: February 14, 2020; Published: February 17, 2020 
cavity, or prior caesarean scar. Intra-myometrial pregnancy is the rarest amongst all the subtypes of ectopic pregnancy, and less than 50 cases have been reported in the literature (less than $1 \%$ of ectopic gestations) [2]. The first case of intramural pregnancy was described by Doderlien, et al. in 1913 [3]. It refers to the uterine conceptus within the myometrium without connection to fallopian tubes, round ligaments, and endometrial cavity.

The etiological factors for intramural ectopic gestation are previous uterine trauma resulting in sinus tract within the endometrium and increased trophoblastic activity \& defective decidualization, which allow the conceptus to penetrate the myometrium, with the most common cause being the former one. The latter is associated with in vitro fertilization and embryo transfer. Others being traumatic causes like caesarean section, manual removal of the placenta, dilatation and curettage $(\mathrm{D} \& \mathrm{C})$ and myomectomy [4].

Intra-myometrial gestation rarely exceeds 12 weeks of gestation. As the gestational sac bulges with the progress of the pregnancy, there is thinning of the myometrium with a risk of uterine rupture and $2.5 \%$ of maternal mortality. Patients remain asymptomatic till uterine rupture. Non-specific symptoms include vaginal spotting, pelvic pain and prolonged menstrual periods. Clinical suspicion rises when urine pregnancy test becomes positive without intracavitary gestational sac. Imaging helps in the diagnosis of the ectopic gestational sac. Transabdominal and Transvaginal ultrasonography helps in the diagnosis of intra-myometrial pregnancy, especially the TVS, which helps in determining the exact location of the gestational sac in the myometrium. MRI helps in the detection of the exact location of the gestational sac and communication between the endometrial cavity \& gestational sac as seen in our case. Serial Beta-HCG is useful in the diagnosis [5]. The diagnosis could be made early even before the rupture by ultrasonography. In our case, both TAS and TVS helped in the diagnosis of the gestational sac in the myometrium. MRI helped in delineating the tract between the endometrial cavity and the gestational sac in the myometrium.

Various conservative techniques like excision by mini laparotomy, hysterotomy by elective laparotomy, excision by operative laparoscopy, diagnostic laparoscopy and medical management by methotrexate are used for the management [6,7]. The advent of all these methods decreased the incidence of laparotomy with hysterectomy which was the only method of treatment until the early 1990s [8]. Rupture of the intra-myometrial pregnancy results in maternal hypovolemic shock and hemo-peritoneum. Prognosis is very poor for intra-myometrial pregnancies.

\section{Conclusion}

If there is a clinical suspicion of ectopic pregnancy, imaging is very much essential to make an appropriate diagnosis. Early diagnosis and proper management of myometrial pregnancy are necessary to prevent fatal prognosis. Transabdominal and transvaginal ultrasonography help in the diagnosis of the ectopic intra-myometrial gestational sac. Delineating the tract can be done with the help of MRI.

\section{References}

1. Larraín D, Marengo F, Bourdel N, Jaffeux P, Aublet-Cuvelier B, et al. (2011) Proximal ectopic pregnancy: A descriptive general population-based study and results of different management options in 86 cases. Feril Steril 95: 867-871.

2. Boukhanni L, Benkaddour YA, Bassir A, Aboulfalah A, Asmouki H, et al. (2014) Case report a rare localization of ectopic pregnancy: Intra-myometrial pregnancy in twin pregnancy following IVF. Case Rep Obstet Gynecol 2014: 10-12.

3. Doederlein TO, Herzog M (1913) A new type of ectopic gestation: Pregnancy in an adenomyoma uteri. Surg Gynecol Obstel 16: 14-20.

4. Marchiolé P, Gorlero F, De Caro G, Podestà M, Valenzano M(2004) Intramural pregnancy embedded in a previous Cesarean section scar treated conservatively. Ultrasound Obstet Gynecol 23: 307-309.

5. Marotta M, Donnez J, Michaux N, Dupont M, Delos M, et al. (2016) Spontaneous intra-myometrial pregnancy mimicking an intramural myoma: A diagnostic challenge. Gynecol Surg 2016: 6-7.

6. Seow KM, Hwang JL, Tsai YL (2001) Ultrasound diagnosis of a pregnancy in a Cesarean section scar. Ultrasound Obstet Gynecol 18: 547-549.

7. Vial Y, Petignat P, Hohlfeld P (2000) Pregnancy in a cesarean scar. Ultrasound Obstet Gynecol 16: 592-593.

8. Falfoul A, Jadoui A, Bellasfar M, Kaabar N, Hamdoun L, et al. (1992) Intramural pregnancy: a case report. J Gynecol Obstet Biol Reprod 21: 641-643.

Copyright: (C2020 Venkatesh M. This is an open-access article distributed under the terms of the Creative Commons Attribution License, which permits unrestricted use, distribution, and reproduction in any medium, provided the original author and source are credited. 